\title{
Effect of graded nerve pressure injuries on motor function
}

\author{
Mika Karasawa, MM, Kumiko Yokouchi, PhD, Akira Kakegawa, PhD, Kyutaro Kawagishi, MD, PhD, \\ Tetsuji Moriizumi, MD, PhD, and Nanae Fukushima, MD, PhD
}

Department of Anatomy, Shinshu University School of Medicine, Matsumoto, Japan

OBJECT The purpose of this study was to determine the minimum amount of nerve fibers required to maintain normal motor function after nerve injury in rats.

METHODS The authors first confirmed that a common peroneal nerve injury caused more aggravating effects on lower limb motor function than tibial nerve injury, as assessed by the static sciatic index (SSI). Thereafter, rats were subjected to varying degrees of crush injury to the common peroneal nerve. At 48 hours after the injury, motor function was assessed using the SSI and slope-walking ability (with slope angles of $30^{\circ}$ and $45^{\circ}$ ). The tibialis anterior muscle, a main muscle innervated by the common peroneal nerve, was removed. Muscle sections were co-labeled with neuronal class III $\beta$-tubulin polyclonal antibody to identify the presence of axons and Alexa Fluor 488-conjugated $\alpha$-bungarotoxin to identify the presence of motor endplates.

RESULTS The evaluation of neuromuscular innervation showed a correlation between SSI scores and ratios of residual axons $\left(r_{s}=0.68, p<0.01\right)$, and there was a statistically significant difference between slope-walking ability and ratios of residual axons $(p<0.01)$. Moreover, the ratios of residual axons in the nerve-crushed rats with normal motor function (SSI above -20 ) ranged from $36.5 \%$ to $88.7 \%$, and those ratios in the success group with slope-walking angles of $30^{\circ}$ and $45^{\circ}$ ranged from $14.7 \%$ to $88.7 \%$ and from $39.8 \%$ to $88.7 \%$, respectively.

CONCLUSIONS In this study of rodents, less than half of the motor axons were sufficient to maintain normal motor function of the lower limb.

http://thejns.org/doi/abs/10.3171/2014.9.JNS141451

KEY WORDS sciatic nerve; common peroneal nerve; crush injury; static sciatic index; slope-walking; peripheral nerve

$\mathrm{T}$ HE authors have previously described the functionally essential neuronal population - the minimum number of neurons related to a specific functionfor some motor and sensory functions by performing nerve-lesioning, tract-lesioning, and neuronal destructive experiments. $4,5,8,9,11,13,14,16$ However, the number of nerve fibers required at the neuromuscular junction to maintain normal motor function is unknown. Therefore, the purpose of this study was to evaluate the extent to which undamaged residual axons contributed to motor function, and to determine the minimum number of nerve fibers required to maintain normal motor function after nerve injury.

Crush injury of the sciatic nerve is widely used in peripheral nerve research. After crush injury of the sciatic nerve, motor function of the lower limb is assessed by the static sciatic index (SSI) for sciatic function. ${ }^{1}$ Several studies have examined sciatic nerve crush injuries and functional assessments using the SSI ${ }^{15,17,18}$ however, it remains unclear which sciatic nerve component (tibial or common peroneal nerve) exerts a marked influence on lower limb motor function assessed by the SSI. We first examined the effect of resection of either the tibial or common peroneal nerve on motor function in rats, and then investigated to what extent undamaged residual axons contributed to preserving motor function in rats that had undergone varying degrees of nerve crush injuries. The number of undamaged residual axons was measured at neuromuscular junctions by immunohistochemically visualized $\beta$ III-tubulin, an axonal marker. The number of acetylcholine receptors was measured in the same sections by histochemically visualized $\alpha$ bungarotoxin, an acetylcholine receptor antagonist. These agents have been widely used to detect nerve terminals and acetylcholine receptors at the neuromuscular junction. ${ }^{6,7,19}$

\section{Methods}

Animals

The experiments were performed on 42 adult female Wistar rats (180-220 g body weight; Japan SLC Inc.). All procedures were conducted in accordance with the NIH 
Guide for the Care and Use of Laboratory Animals, and the protocols were approved by our Institutional Animal Care and Use Committee. All efforts were made to minimize animal suffering and pain. Surgical procedures were performed under general anesthesia induced with an intraperitoneal injection of a mixture of pentobarbital (50 $\mathrm{mg} / \mathrm{kg}$ ) and medetomidine $(10 \mathrm{mg} / \mathrm{kg})$. Atipamezole hydrochloride $(2 \mathrm{mg} / \mathrm{kg})$ was injected intraperitoneally to reverse the effect of anesthesia.

\section{Resection of the Tibial Nerve or Common Peroneal Nerve and Functional Assessment Using SSI}

The left sciatic nerve and its 2 major branches, the tibial and common peroneal nerves, were exposed through a gluteal muscle-splitting incision. The tibial $(\mathrm{n}=2)$ or common peroneal $(n=2)$ nerve was resected $1 \mathrm{~cm}$ in length just before entering the crural muscle. After surgery, rats were housed under standard laboratory conditions with a 12-hour light/dark cycle and room temperature maintained at $22^{\circ} \mathrm{C}$. Food and water were supplied ad libitum. To assess functional loss following nerve resection, we performed a conventional footprint analysis using procedures described in previous publications.,10-12,20,21 Briefly, footprints were obtained 24 and 48 hours after nerve resection to assess the effects of nerve injury in the acute period. Hind footprints were obtained by applying quick-drying Indian ink to the hind feet and allowing the animals to walk freely in a box $(70.0 \times 33.5 \times 22.0 \mathrm{~cm})$; thus tracks were left on the underlying paper. Two parameters of 1-5 toe spread and 2-4 toe spread lengths on both sides were measured manually by a ruler and SSI was calculated according to the formula defined by Bervar. ${ }^{1}$ When footprints could not be obtained because of very severe motor dysfunction, the SSI score was considered to be -100.0 . SSI scores were calculated for each animal from 15 sets of footprints, and the trimmed mean (i.e., the average with the top 3 and bottom 3 scores excluded) was considered the best estimate for each animal.

\section{Graded Pressure Injuries of the Common Peroneal Nerve}

The left common peroneal nerve $(n=32)$ was exposed and crushed with either a brain aneurysm clip (Sugita standard aneurysm clip, holding force $147 \mathrm{~g}$; Mizuho Ikakogyo) for 1, 2, 3, 4, 5, and 10 seconds, or a disposable microvascular clip (holding force $60 \mathrm{~g}$; BEAR Medic Corporation) for $1,20,60,80,90,100,110,120$, and $180 \mathrm{sec}-$ onds. Because the brain aneurysm clip caused strong damage (SSI score < -80) in a very short time due to its strong holding force, crush injuries were also performed using a weaker microvascular clip. The left common peroneal nerve was exposed but left intact in the control rats $(n=6)$.

\section{Functional Assessment Using SSI and Slope-Walking Ability}

At 48 hours after surgery, footprint analyses were performed for all rats in the control and nerve-crushed groups using the procedures described above. After motor function was assessed with SSI, the control and nerve-crushed rats were placed on the $30^{\circ}$ and $45^{\circ}$ slopes constructed with a plastic panel $(50 \times 30 \mathrm{~cm})$ to evaluate slope-walking ability. Before placing the rat on the slope, both hind feet were wiped with a wet towel and examined to determine whether the rat could maneuver in both of the slope angles. A trial was considered a success if the rat could walk up the slope or stay on the slope for at least 5 seconds.

\section{Tissue Preparation}

After motor function assessments, rats were killed with an intraperitoneal injection of sodium pentobarbital (100 $\mathrm{mg} / \mathrm{kg}$ ) and perfused through the heart with $300 \mathrm{ml}$ of $4 \%$ paraformaldehyde in $0.1 \mathrm{M}$ phosphate buffer. The tibialis anterior muscle on the nerve-injured side was removed, postfixed overnight in fixative, and then soaked in 30\% phosphate-buffered sucrose for 2 days. The muscles were cut longitudinally into $50-\mu \mathrm{m}$-thick serial sections on a freezing microtome and collected at 600- $\mu$ m intervals. To evaluate the neuromuscular innervation, the sections were co-labeled with neuronal class III $\beta$-tubulin polyclonal antibody (Covance) to identify the presence of axons and Alexa Fluor 488-conjugated $\alpha$-bungarotoxin (Life Technologies) to identify the presence of motor endplates. ${ }^{6,7}$ The sections were immersed for 2 hours in phosphate-buffered saline containing $0.3 \%$ Triton $\mathrm{X}-100$, and then incubated at room temperature for 2 days in rabbit anti- $\beta$ III-tubulin antibody (1:25000). The sections were then incubated for 5 hours in a mixture of Alexa Fluor 546-conjugated antirabbit immunoglobulin G (1:1000, Life Technologies) and Alexa Fluor 488-conjugated $\alpha$-bungarotoxin (1:2000). The sections were rinsed, mounted on coated slides, and coverslipped with FluorSave Reagent (Calbiochem).

\section{Quantification of Undamaged Residual Axons and Statistical Analysis}

The ratio of the number of $\beta$ III-tubulin-positive nerve terminals to $\alpha$-bungarotoxin-positive acetylcholine receptors was determined for both control and nerve-crushed rats using a fluorescence microscope (Nikon); the ratio of undamaged residual axons to acetylcholine receptors in each nerve-crushed rat was expressed as a percentage of the mean value of the control. Z-stack images were captured at 2.5- $\mu \mathrm{m}$ intervals using a BZ-9000 fluorescence microscope (Keyence) to view the images of thick samples. The data were expressed by means \pm standard deviations (SD), and were compared using the Student t-test and Spearman's correlation analysis. Those $\mathrm{p}$ values less than 0.05 were considered statistically significant.

\section{Results}

\section{Resection of the Tibial or Common Peroneal Nerve}

Figure 1 shows the changes in SSI scores for tibial and common peroneal nerve-resected rats. SSI scores for the tibial nerve-resected rats were -34.2 and -35.9 at 24 hours, and -32.2 and -47.1 at 48 hours after nerve resection. The common peroneal nerve-resected rats, however, could not place the sole of the foot on the floor and their SSI scores were -100.0 and -100.0 both at 24 and 48 hours after nerve resection.

\section{Graded Nerve Pressure Injuries and SSI Scores}

Because the nerve lesion experiments confirmed that 


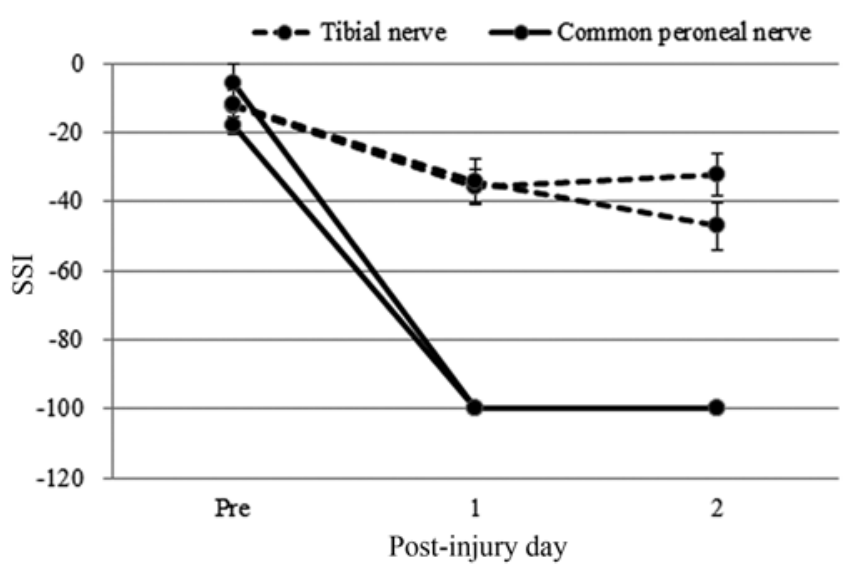

FIG. 1. SSI scores in the tibial nerve-resected $(n=2)$ and common peroneal nerve-resected rats $(n=2)$ at 1 and 2 days after injury.

the common peroneal nerve exerted a stronger influence on lower limb motor function than the tibial nerve, rats were subjected to varying degrees of crush injury to the common peroneal nerve. The 6 control rats showed no gait disturbances, and their SSI scores were above -20 (range -1.3 to -18.3 ), the standard SSI range for normal motor function. Rats subjected to varying degrees of common peroneal nerve-crush injuries showed SSI scores that ranged from 1.7 to -100.0 (Table 1). Figure 2 shows representative footprints taken from nerve-crushed rats. Based on the SSI scores, we classified nerve-crushed rats into 4 groups that demonstrated normal (score above $-20, n=17$; Fig. 2A), mildly disordered (score -20 to $-50, \mathrm{n}=7$; Fig. $2 \mathrm{~B}$ ), moderately disordered (score -50 to $-80, \mathrm{n}=3$; Fig. $2 \mathrm{C}$ ), or severely disordered (score below $-80, \mathrm{n}=5$; Fig. 2D) motor functions.

\section{Graded Nerve Pressure Injuries and Undamaged Residual Axons}

Figure 3 shows neuromuscular junctions of tibialis anterior muscle fibers in both control and common peroneal nerve-crushed rats. Neuromuscular junctions that had both $\beta$ III-tubulin and $\alpha$-bungarotoxin staining were considered innervated, whereas neuromuscular junctions stained with only $\alpha$-bungarotoxin were considered denervated. In the control rats, the ratio of the number of $\beta$ III-tubulin-positive nerve terminals to $\alpha$-bungarotoxinpositive acetylcholine receptors was $91.9 \% \pm 2.7 \%$ (858 \pm 62 terminals/935 \pm 77 receptors). The ratios for the nervecrushed rats with normal, mildly disordered, moderately disordered, and severely disordered motor functions were $62.1 \% \pm 13.0 \%$ (612 \pm 148 terminals/982 \pm 95 receptors $)$, $53.9 \% \pm 13.6 \%$ (538 \pm 121 terminals/1007 \pm 90 receptors), $47.0 \% \pm 12.5 \%$ (532 \pm 138 terminals/1133 \pm 14 receptors $)$, and $9.3 \% \pm 3.8 \%(93 \pm 37$ terminals/1004 \pm 66 receptors $)$, respectively. The ratios of residual axons to acetylcholine receptors that were expressed as a percentage of the control $(91.9 \% \pm 2.7 \%, \mathrm{n}=6)$ in the nerve-crushed rats with normal, mildly disordered, moderately disordered, and severely disordered motor functions were calculated as $67.6 \% \pm 14.2 \%, 58.6 \% \pm 14.8 \%, 51.2 \% \pm 13.6 \%$, and $10.2 \%$ $\pm 4.1 \%$, respectively. The ratios of residual axons to ace-
TABLE 1. Results of assessments of motor function and ratios of undamaged residual axons

\begin{tabular}{|c|c|c|c|c|c|c|}
\hline \multirow[b]{2}{*}{ Clip Type } & \multirow{2}{*}{$\begin{array}{c}\text { Animal } \\
\text { No. }\end{array}$} & \multirow{2}{*}{$\begin{array}{c}\text { Duration } \\
\text { (sec) }\end{array}$} & \multirow[b]{2}{*}{ SSI } & \multicolumn{2}{|c|}{$\begin{array}{c}\text { Slope- } \\
\text { Walking }\end{array}$} & \multirow{2}{*}{$\begin{array}{c}\text { Residua } \\
\text { Axons } \\
\text { (\% of } \\
\text { Control }\end{array}$} \\
\hline & & & & $30^{\circ}$ & $45^{\circ}$ & \\
\hline \multicolumn{7}{|c|}{ Brain aneurysm } \\
\hline & 1 & 1 & -2.1 & $S$ & $S$ & 65.9 \\
\hline & 2 & 1 & -10.4 & $S$ & $S$ & 66.5 \\
\hline & 3 & 2 & -5.9 & $S$ & $S$ & 39.8 \\
\hline & 4 & 2 & -22.9 & $S$ & $\mathrm{~F}$ & 55.1 \\
\hline & 5 & 3 & -30.8 & $S$ & $S$ & 49.9 \\
\hline & 6 & 3 & -100.0 & $\mathrm{~F}$ & $\mathrm{~F}$ & 3.7 \\
\hline & 7 & 4 & -19.2 & $S$ & $S$ & 87.8 \\
\hline & 8 & 4 & -20.7 & $S$ & $S$ & 49.4 \\
\hline & 9 & 4 & -22.1 & $S$ & $S$ & 81.7 \\
\hline & 10 & 4 & -54.9 & $S$ & $S$ & 56.6 \\
\hline & 11 & 4 & -59.8 & $S$ & $S$ & 61.2 \\
\hline & 12 & 4 & -75.0 & $\mathrm{~F}$ & $\mathrm{~F}$ & 35.7 \\
\hline & 13 & 5 & -100.0 & $\mathrm{~F}$ & $\mathrm{~F}$ & 9.1 \\
\hline & 14 & 10 & -91.7 & $\mathrm{~F}$ & $\mathrm{~F}$ & 11.0 \\
\hline \multicolumn{7}{|l|}{ Microvascular } \\
\hline & 15 & 1 & -4.9 & S & S & 63.1 \\
\hline & 16 & 1 & -7.8 & S & $\mathrm{F}$ & 62.7 \\
\hline & 17 & 20 & 1.7 & $S$ & $S$ & 73.0 \\
\hline & 18 & 60 & -12.7 & $S$ & $\mathrm{~F}$ & 36.5 \\
\hline & 19 & 80 & -1.9 & $S$ & $S$ & 66.5 \\
\hline & 20 & 90 & -5.4 & $S$ & $S$ & 86.8 \\
\hline & 21 & 90 & -29.9 & $S$ & $S$ & 50.3 \\
\hline & 22 & 100 & -11.6 & $S$ & $S$ & 88.7 \\
\hline & 23 & 100 & -39.6 & $S$ & $S$ & 45.9 \\
\hline & 24 & 100 & -94.7 & $\mathrm{~F}$ & $\mathrm{~F}$ & 12.3 \\
\hline & 25 & 110 & -0.5 & $S$ & $S$ & 75.5 \\
\hline & 26 & 110 & -3.0 & $S$ & $S$ & 69.9 \\
\hline & 27 & 110 & -4.8 & $S$ & $S$ & 72.3 \\
\hline & 28 & 110 & -30.7 & $S$ & $S$ & 78.0 \\
\hline & 29 & 120 & -90.3 & $S$ & $\mathrm{~F}$ & 14.7 \\
\hline & 30 & 180 & -4.5 & $S$ & $S$ & 70.3 \\
\hline & 31 & 180 & -5.9 & $S$ & $S$ & 60.2 \\
\hline & 32 & 180 & -19.5 & $S$ & $S$ & 63.8 \\
\hline
\end{tabular}

$F=$ failure $;=$ success .

tylcholine receptors (percentage of control) for individual animals in the nerve-crushed rats are shown in Table 1.

\section{Correlation Analysis of SSI and Undamaged Residual Axons}

In Fig. 4, SSI scores are plotted against the ratios of residual axons to acetylcholine receptors (percentage of control) for common peroneal nerve-crushed rats. Statistically significant positive correlations were observed between an increasing SSI score and an increasing ratio of residual axons $\left(r_{s}=0.68, p<0.01\right)$. 


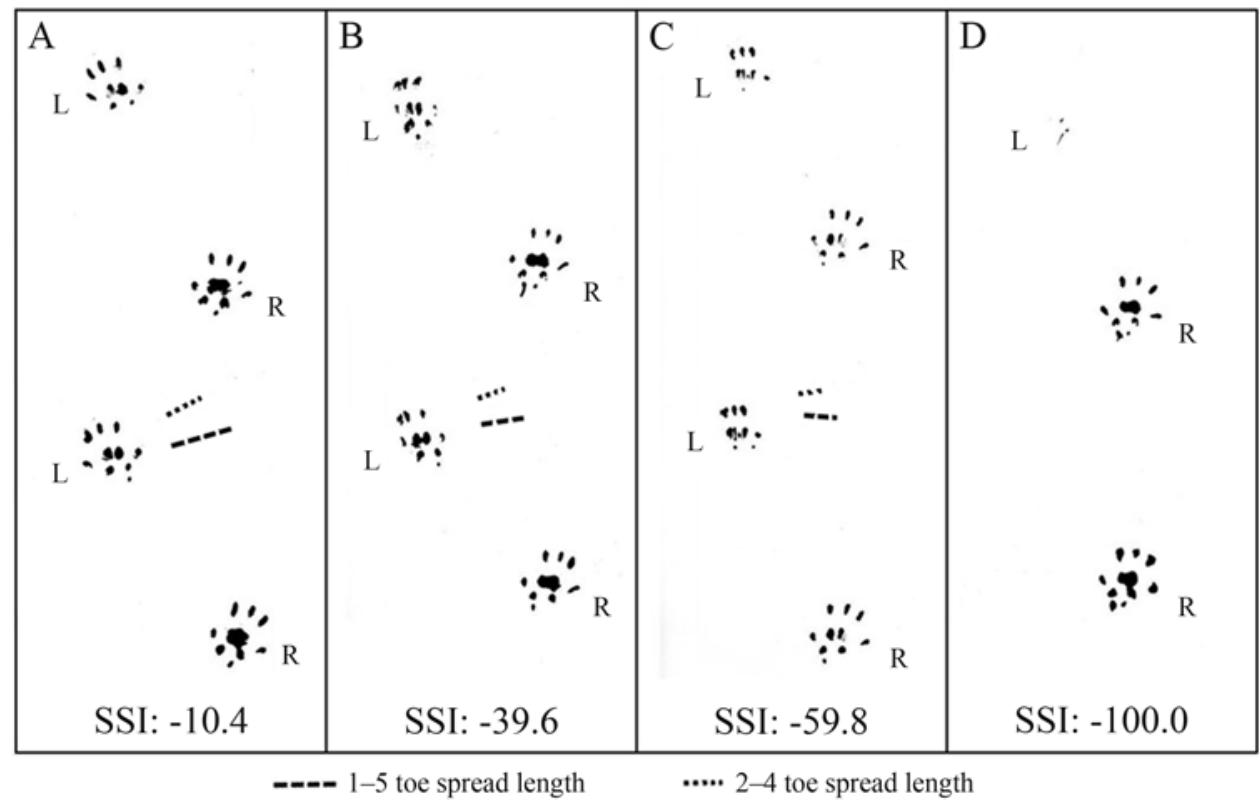

FIG. 2. Representative footprints of the nerve-crushed rats with normal (A), mildly disordered (B), moderately disordered (C), and severely disordered (D) motor functions. SSI scores are denoted on the figure.

\section{Slope-Walking Ability}

The control rats successfully performed the slopewalking test at both $30^{\circ}$ and $45^{\circ}$ angles. The common peroneal nerve-crushed rats were divided into 2 groups based on whether they succeeded or failed in the slopewalking test. Figure 5 shows the ratios of residual axons to acetylcholine receptors (percentage of control) in the success and failure groups, which were (means \pm SDs): $62.7 \% \pm 16.9 \%$ and $14.4 \% \pm 12.4 \%$ at $30^{\circ}$, and $66.2 \% \pm$ $13.6 \%$ and $26.8 \% \pm 21.6 \%$ at $45^{\circ}$. The differences between the 2 groups were statistically significant for both angles tested $(\mathrm{p}<0.01)$.

\section{Discussion}

\section{Effect of Tibial and Common Peroneal Nerve Resection on Motor Function}

The SSI scores obtained after nerve resection revealed that motor dysfunction was more severe in rats with common peroneal nerve resections than those with tibial nerve resections. That is, the effect of nerve resection on motor function as assessed using SSI was greater for common peroneal nerve injury than for tibial nerve injury. Rats that underwent common peroneal nerve resection received an SSI score of -100.0 (severely disordered motor function), whereas rats that underwent tibial nerve resection received an SSI score of approximately -40.0 (mildly disordered motor function). The common peroneal nerve innervates the ankle dorsiflexors, tibialis anterior, and extensor digitorum longus muscles; the tibial nerve innervates the plantar flexors, gastrocnemius, and soleus muscles. ${ }^{2,3,22}$ The common peroneal nerve-resected rats showed severe motor dysfunction due to a lack of ankle dorsiflexion and toe extension (i.e., drop foot). Ankle dorsiflexion appeared to be very important during walking in the nerve-injured rats.

\section{Graded Nerve Pressure Injuries}

To produce varying degrees of crush injuries to the common peroneal nerve, we used 2 different clips (the brain aneurysm clip and microvascular clip) and varied the durations for which the clips were attached to the nerve i.e., $1,2,3,4,5$, and 10 seconds for the brain aneurysm clip and $1,20,60,80,90,100,110,120$, and 180 seconds for the microvascular clip. After the crush injury, approximately half of the nerve-injured rats showed no gait disturbances despite nerve crushing. Rats with injuries produced by the brain aneurysm clip showed motor dysfunctions that varied with the duration of the crush injury. Their SSI scores and ratios of residual axons to acetylcholine receptors ranged from -2.1 to -22.9 and from $39.8 \%$ to $66.5 \%$ for $1-2$ seconds, from -19.2 to -100.0 and from $3.7 \%$ to $87.8 \%$ for 3-4 seconds, and from -91.7 to -100.0 and from $9.1 \%$ to $11.1 \%$ for more than 5 seconds. Conversely, there was no relationship between motor dysfunction and the duration of crush injuries produced with the microvascular clip. For example, the rats subjected to crush injury for 100 seconds showed different motor dysfunctions, and their SSI scores ranged from -11.6 (normal motor function) to -94.7 (severely disordered motor function). Furthermore, the rats that were subjected to crush injury for 180 seconds showed normal motor function (SSI above -20). Therefore, it appears that the motor dysfunctions in rats with a nerve crush injury produced with the microvascular clip were related to the amount of connective tissues around the nerve because the pressure exerted by this clip was relatively weak. Because great care was taken not to damage the nerve itself before the nerve crushing procedure, the total removal of the perineural connective tissue appeared impossible, and thus varying amounts of the connective tissue inevitably remained around the nerve, which caused differential deteriorating effects on the nerve among rats with the same 

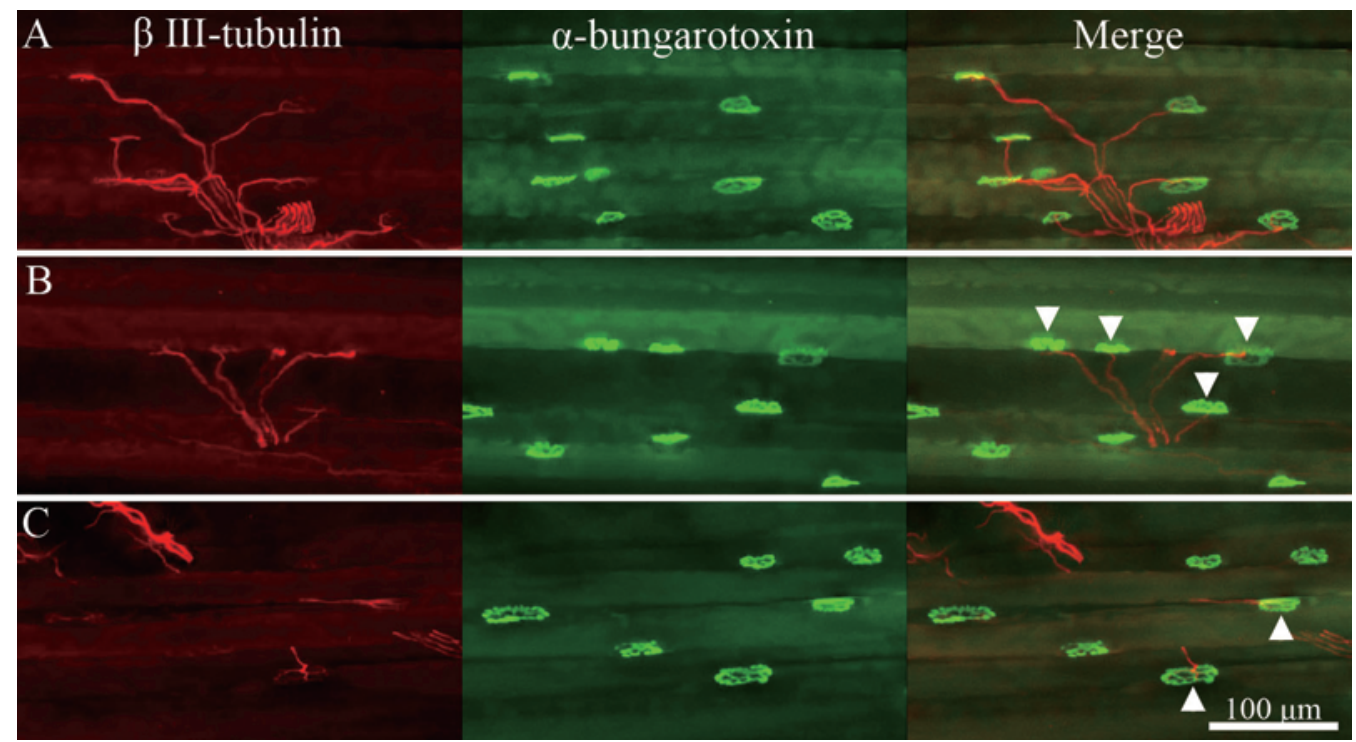

FIG. 3. Immunofluorescence staining of tibialis anterior muscle sections with an anti- $\beta$ III-tubulin antibody and $\alpha$-bungarotoxin, as well as an overlay of $\beta$ III-tubulin and $\alpha$-bungarotoxin images (merge). A: Images obtained in a control rat. The ratio of the number of $\beta$ III-tubulin-positive nerve terminals to that of $\alpha$-bungarotoxin-positive acetylcholine receptors was $97.1 \%$. B and C: Images obtained in nerve-crushed rats. The ratios of the number of nerve terminals to that of acetylcholine receptors were $56.2 \%(B)$ and $36.6 \%$ (C). Arrowheads indicate neuromuscular junctions stained with both markers. Figure is available in color online only.

duration of crush injuries. Taken together, the varying degrees of nerve crush injury were successfully executed to the common peroneal nerve using 2 different clips, as is indicated by a wide range of SSI scores (from 1.7 to -100.0) and ratios of residual axons (from $3.7 \%$ to $88.7 \%$ ).

\section{Undamaged Residual Axons to Maintain Normal Motor Function}

The slope-walking test, which aims to assess motor function, was used for the first time in this study. Slopewalking ability might reflect motor dysfunction because rats require ankle dorsiflexion to perform these movements. According to the functional assessments conduct-

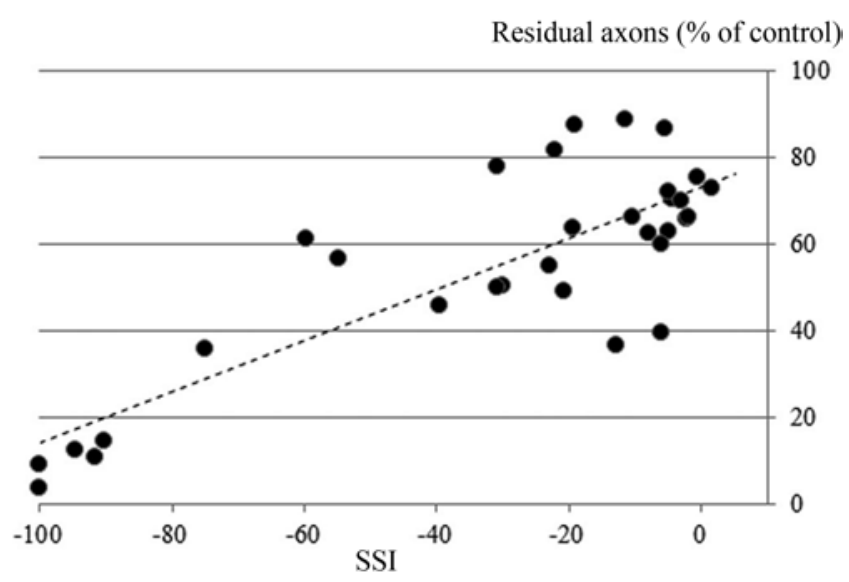

FIG. 4. Graph showing linear relationships between SSI scores and ratios of undamaged residual axons of the experimental rats with varying degrees of crush injury $\left(r_{s}=0.68, p<0.01\right)$. Each dot represents 1 animal, but some dots overlap. ed using SSI and slope-walking ability following varying degrees of crush injuries, there was a significant positive correlation between motor function and the number of undamaged residual axons. In our previous study, we demonstrated that a functionally essential neuronal population exists for a specific neural function. $4,5,8,9,11,13,14,16$ Similarly, concerning axonal components essential for normal motor function, approximately $40 \%$ of undamaged axons appear to be sufficient for normal motor function in rats with a common peroneal nerve-crush injury because the ratios of residual axons to acetylcholine receptors in the nervecrushed rats with normal motor function (SSI above -20) ranged from $36.5 \%$ to $88.7 \%$, and the ratios for the success group in the slope-walking test at angles of $30^{\circ}$ and $45^{\circ}$
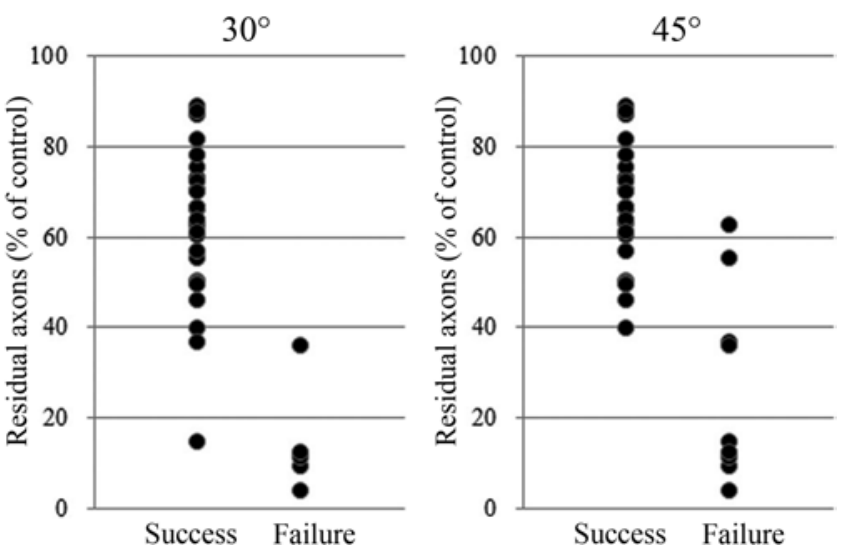

FIG. 5. Ratios of undamaged residual axons of the experimental rats with varying degrees of crush injury for slope-walking angles of $30^{\circ}$ (left) and $45^{\circ}$ (right). Each dot represents 1 animal, but some dots in each group overlap. 
ranged from $14.7 \%$ to $88.7 \%$ and from $39.8 \%$ to $88.7 \%$, respectively. However, for some nerve-injured rats, there were inconsistencies between motor function and the ratio of undamaged residual axons to acetylcholine receptors. Some rats with more than $40 \%$ of undamaged axons showed mild or moderate motor dysfunction and failed the slope-walking test at an angle of $45^{\circ}$. This inconsistency might have been caused by the method that we used to assess the ratio of residual axons to acetylcholine receptors only in the tibialis anterior muscle, one of the primary muscles innervated by the common peroneal nerve.

\section{Study Limitations}

The limitations of this study include the measurement of motor function. Functional assessments were performed only by 2 methods, the SSI and slope-walking ability. The different values might be obtained from other assessments such as video assessment and ankle kinematics. Moreover, an appropriate evaluation method needs to be chosen if a similar experiment is performed in other models of nerve injury.

\section{Conclusions}

Based on the results of this study, we conclude that less than half of the motor axons can suffice to maintain normal motor function of the lower limb in rats.

\section{References}

1. Bervar M: Video analysis of standing-an alternative footprint analysis to assess functional loss following injury to the rat sciatic nerve. J Neurosci Methods 102:109-116, 2000

2. Chen B, Song Y, Liu Z: Promotion of nerve regeneration in peripheral nerve by short-course FK506 after end-to-side neurorrhaphy. J Surg Res 152:303-310, 2009

3. Degens H, Meessen NE, Wirtz P, Binkhorst RA: The development of compensatory hypertrophy in the plantaris muscle of the rat. Ann Anat 177:285-289, 1995

4. Fukushima N, Oikawa S, Yokouchi K, Kawagishi K, Moriizumi T: The minimum number of neurons in the central olfactory pathway in relation to its function: a retrograde fiber tracing study. Chem Senses 27:1-6, 2002

5. Fukushima N, Yokouchi K, Kawagishi K, Kakegawa A, Sumitomo N, Karasawa M, et al: Quantitative analysis of survival of hypoglossal neurons in neonatally nerve-injured rats: correlation with milk intake. Arch Oral Biol 59:616-620, 2014

6. Grosheva M, Guntinas-Lichius O, Angelova SK, Kuerten S, Alvanou A, Streppel M, et al: Local stabilization of microtubule assembly improves recovery of facial nerve function after repair. Exp Neurol 209:131-144, 2008

7. Guntinas-Lichius O, Irintchev A, Streppel M, Lenzen M, Grosheva M, Wewetzer K, et al: Factors limiting motor recovery after facial nerve transection in the rat: combined structural and functional analyses. Eur J Neurosci 21:391402, 2005

8. Higashiyama F, Fukushima N, Yokouchi K, Kawagishi K, Moriizumi T: The minimum number of facial motor neurons essential for minimal whisker movement in neonatally nerve-transected young adult rats. J Neurosurg 102 (4 Suppl):390-395, 2005

9. Hirayama S, Kawagishi K, Yokouchi K, Fukushima N, Karasawa M, Moriizumi T: Regenerative capacity of bulbar projection neurons during development: a quantitative neuronal analysis with functional correlation. Chem Senses 39:47-56, 2014
10. Itsubo T, Fukushima N, Kakegawa A, Yokouchi K, Kawagishi K, Kato H, et al: Effects of repeated crush injuries on motor functional recovery of the sciatic nerve. Neurol Res 34:908-914, 2012

11. Kakegawa A, Yokouchi K, Fukushima N, Fukuyama T, Moriizumi T: Motor neurons essential for normal sciatic function in neonatally nerve-injured rats. Neuroreport 17:1149-1152, 2006

12. Kakegawa A, Yokouchi K, Itsubo T, Kawagishi K, Karasawa $\mathrm{M}$, Moriizumi T, et al: Correlation between motor function and axonal morphology in neonatally sciatic nerve-injured rats. Anat Sci Int [epub ahead of print], 2014

13. Kawagishi K, Yokouchi K, Fukushima N, Sakamoto M, Sumitomo N, Moriizumi T: Determination of functionally essential neuronal population of the olfactory epithelium for nipple search and subsequent suckling behavior in newborn rats. Brain Res 1276:50-57, 2009

14. Kobayashi S, Koyama J, Yokouchi K, Fukushima N, Oikawa S, Moriizumi T: Functionally essential neuronal population of the facial motor nucleus. Neurosci Res 45:357-361, 2003

15. Korte N, Schenk HC, Grothe C, Tipold A, Haastert-Talini $\mathrm{K}$ : Evaluation of periodic electrodiagnostic measurements to monitor motor recovery after different peripheral nerve lesions in the rat. Muscle Nerve 44:63-73, 2011

16. Koyama J, Yokouchi K, Fukushima N, Kawagishi K, Moriizumi T: Great potentiality of neonatal facial motor neurons for neural plasticity as determined by functionally essential neuronal population. Neurosci Res 46:85-93, 2003

17. Mensching L, Börger AK, Wang X, Charalambous P, Unsicker K, Haastert-Talini K: Local substitution of GDF-15 improves axonal and sensory recovery after peripheral nerve injury. Cell Tissue Res 350:225-238, 2012

18. Mohammadi R, Mahmoodi H: Improvement of peripheral nerve regeneration following nerve repair by silicone tube filled with curcumin: a preliminary study in the rat model. Int J Surg 11:819-825, 2013

19. Pitman MJ, Berzofsky CE, Alli O, Sharma S: Embryologic innervation of the rat laryngeal musculature-a model for investigation of recurrent laryngeal nerve reinnervation. Laryngoscope 123:3117-3126, 2013

20. Varejão AS, Cabrita AM, Patrício JA, Bulas-Cruz J, Gabriel RC, Melo-Pinto P, et al: Functional assessment of peripheral nerve recovery in the rat: gait kinematics. Microsurgery 21:383-388, 2001

21. Varejão AS, Meek MF, Ferreira AJ, Patrício JA, Cabrita AM: Functional evaluation of peripheral nerve regeneration in the rat: walking track analysis. J Neurosci Methods 108:1-9, 2001

22. Waters HJ, Barnett G, O'Hanlon GM, Lowrie MB: Motoneuron survival after neonatal peroneal nerve injury in the ratevidence for the sparing effect of reciprocal inhibition. Exp Neurol 152:95-100, 1998

\section{Author Contributions}

Conception and design: Fukushima, Karasawa, Moriizumi. Acquisition of data: Fukushima, Karasawa, Yokouchi, Kakegawa. Analysis and interpretation of data: Fukushima, Karasawa, Kawagishi, Moriizumi. Drafting the article: Fukushima, Karasawa, Moriizumi. Critically revising the article: all authors. Reviewed submitted version of manuscript: all authors. Approved the final version of the manuscript on behalf of all authors: Fukushima.

\section{Correspondence}

Nanae Fukushima, Department of Anatomy, Shinshu University School of Medicine, 3-1-1 Asahi, Matsumoto, Nagano 390-8621, Japan.email: nanae@shinshu-u.ac.jp. 